\title{
Prevalência e fatores associados à sintomatologia depressiva em idosos residentes no Nordeste do Brasil
}

\author{
Prevalence and associated factors of depressive simptomatology in elderly \\ residents in the Northeast of Brazil
}

\author{
Álvaro Campos Cavalcanti Maciel e Ricardo Oliveira Guerra
}

\begin{abstract}
Resumo
Objetivo: Analisar a influência de fatores sociodemográficos, de saúde física, capacidade funcional e função cognitiva sobre a sintomatologia depressiva de idosos do município de Santa Cruz, no Rio Grande do Norte. Métodos: Estudo com delineamento transversal de base populacional, incluindo 310 idosos, acima de 60 anos, residentes na zona urbana da cidade, nos quais se aplicou a Escala de Depressão Geriátrica (GDS-15). A análise estatística foi realizada com nível de significância $p=0,05$, com cálculo da respectiva odds ratio (OR) na regressão logística binária. Resultados: Encontrou-se uma prevalência de $25,5 \%$ de sujeitos considerados casos de depressão, nos quais, a partir de análise multivariada, verificou-se associação significativa com idade acima de 75 anos $(p=0,046)$, analfabetismo $(p=0,037)$, má percepção de saúde $(p<0,001)$ e dependência para atividades instrumentais da vida diária (AIVD) $(p=0,001)$. Conclusões: As variáveis idade acima de 75 anos, analfabetismo, má percepção de saúde e dependência para AIVD estiveram associadas de forma independente à presença de sintomatologia depressiva nos idosos da nossa população. Os autores discutem que a identificação de fatores que influenciam o surgimento de sintomas depressivos em idosos constitui passo fundamental para o planejamento das ações que visem reduzir os efeitos dessa enfermidade na qualidade de vida dessas pessoas.
\end{abstract}

Palavras-chave: idoso, depressão, envelhecimento.

\begin{abstract}
Objective: To analyze the influence of socio-demographic factors, physical health, functional status and cognitive function on depressive symptomatology of the elderly from Santa Cruz, Rio Grande do Norte. Methods: A cross-sectional study was conducted in a sample constituted by 310 elderly above 60 old years who live in an urban zone of the town in which the Geriatric Depression Scale (GDS-15) was applied. The statistical analysis was carried out with significance level $p=0.005$, and the respective odds ratio (OR) was calculated in the binary logistic regression. Results: A prevalence of $25.5 \%$ of subjects considered cases of depression was found, and after a multivariate analysis significant association with age over 75 years $(p=0.046)$, illiteracy $(p=0.037)$, poor health perception $(p<0.001)$ and dependency for instrumental activities of daily living $(p=0.001)$ was verified. Conclusions: The variables age above 75 years, illiteracy, bad health perception and being dependent in basic activities of daily living were independently associated with the presence of depressive symptoms in this population. The authors suggest that the identification of factors that influence the onset of depressive general situations in the elderly is a fundamental step to plan actions to reduce the effects of this condition on these people's living quality.
\end{abstract}

Key words: elderly, depression, aging.

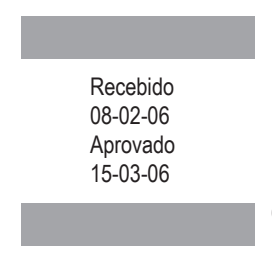

Departamento de Fisioterapia da Universidade Federal do Rio Grande do Norte (UFRN)

(Maciel ACC, Guerra RO)

Hospital Universitário Ana Bezerra/UFRN (Maciel ACC)

Correspondência para: Álvaro Campos Cavalcanti Maciel

Rua Moises Gosson, 1.442 - Lagoa Nova - 59056-060 - Natal-RN - Tel.: (84) 3215-4270

- e-mail: alvarohuab@ig.com.br 


\section{Introdução}

Entre as várias alterações neuropsiquiátricas que acometem os idosos, a depressão assume papel de destaque pelo forte impacto no sistema de saúde e sua alta prevalência nessa população (Spear, 2002; Barcia e Alcântara, 2000). Embora isso também seja verídico em outras faixas etárias adultas, a depressão permanece um problema significativo encontrado por profissionais que trabalham com o idoso (Guccione, 2002). Sobre a prevalência desses quadros em meio aos idosos, diversos estudos apontam para valores entre $15 \%$ e 30\%, de acordo com local de moradia, situação socioeconômica e escala utilizada para a detecção dos casos (Chattat et al., 2001; Barbosa et al., 2005).

A presença desse quadro em idosos desperta um especia interesse por sua transcendência para a saúde, capacidade funcional e os distintos aspectos psicossociais dessa população. Existe, nos estudos atuais, uma grande controvérsia sobre o desenvolvimento da depressão em populações idosas, pois a apresentação de seus sinais e sintomas nesse tipo de coletivo resulta na dificuldade em diferenciar o que podemos considerar envelhecimento normal dos processos patológicos. Confundir os sintomas de depressão com enfermidades somáticas e ansiedade é comum em qualquer grupo de idade, mas parece ser um problema especial em idosos, principalmente nos mais frágeis (Steffens, 2005).

Alguns autores afirmam que a aparente associação entre avanço da idade e aumento do risco de desenvolvimento da demência e da depressão está relacionada às mudanças na estrutura do córtex cerebral (Rozenthal et al., 2004). Na atualidade, a etiologia da depressão é vista de forma ampla e integrada, dentro de um contexto de inter-relação de numerosos problemas psicológicos, sociais e físicos que muitas vezes mascaram o diagnóstico e dificultam 0 tratamento (Snowdon, 2002; Steffens, 2005).

Além disso, os transtornos depressivos levam a uma importante repercussão sobre a qualidade de vida dos idosos, razão pela qual sua detecção e seu tratamento precoce podem contribuir favoravelmente para melhorar suas atitudes diante da enfermidade e, principalmente, melhorar seu bem-estar (Barbosa et al., 2005).

Em virtude dos fatos, o conhecimento dessa condição e dos fatores a ela associados constitui um passo essencial no processo de planejamento das ações de saúde, pois pode contribuir para diminuição de internações hospitalares e uso de medicamentos, melhora do estado funcional e, por fim, redução dos gastos sanitários.

Sendo assim, este estudo objetiva analisar que variáveis associam-se à presença de depressão em idosos que vivem na comunidade, contribuindo, portanto, para o seu entendimento e favorecendo o planejamento de condutas eficazes para esses indivíduos.

\section{Métodos}

Foi realizado um estudo transversal na cidade de Santa Cruz, localizada a $120 \mathrm{~km}$ de Natal, capital do Rio Grande do Norte, Região Nordeste do Brasil, no período de julho a novembro de 2002. Enviado ao Comitê de Ética em Pesquisa local, o trabalho foi submetido a avaliação e aprovado, de acordo com o parecer $84 / 02$. Essa aprovação garantiu o respeito à dignidade humana $\mathrm{e}$ 0 desenvolvimento da pesquisa dentro dos padrões éticos. Além disso, todos os sujeitos assinaram o termo de consentimento livre $e$ esclarecido, onde constavam todas as informações relevantes sobre sua participação no estudo.

A amostra foi composta por 310 idosos com 60 anos ou mais, residentes em domicílios na zona urbana de Santa Cruz e cadastrados no Sistema de Informações da Atenção Básica (SIAB), em dezembro de 2001, escolhidos de forma probabilística aleatória sistemática. Aquele valor correspondeu a $10,09 \%$ da população inicial de 3.070 idosos.

Levando em consideração a heterogeneidade do município e buscando a representatividade da amostra, foram sorteados os bairros Paraíso, Conjunto Cônego Monte (CCM) e Centro, que representaram o pior, o intermediário e o melhor estrato socioeconômico. Por buscar a prevalência de idosos com depressão, todos os indivíduos selecionados aleatoriamente foram avaliados, independentemente de sua condição física ou mental. Todas as entrevistas foram conduzidas nos domicílios dos idosos.

Para a avaliação foi elaborado um questionário multidimensional que constou dos seguintes itens:

- aspectos sociodemográficos: idade, sexo, cor, escolaridade, estado civil, atividade laboral, atividade nas horas livres e tamanho da família;

- aspectos de saúde física: autopercepção de saúde, patologias (diabetes mellitus, hipertensão arterial sistêmica, doenças pulmonares, fratura de quadril, reumatismo, déficits visual e auditivo e câncer), medicamentos em uso contínuo e número de internamentos no último ano;

- capacidade funcional: atividades básicas da vida diária (ABVD) pelo índice de Katz (Katz et al., 1963) e atividades instrumentais da vida diária (AIVD) pelo índice de Lawton (Lawton e Brody, 1969);

- aspectos de saúde mental: na avaliação da presença de sintomatologia depressiva foi utilizada a Escala de Depressão Geriátrica (GDS-15) (Shah et al., 1997), com o ponto de corte padrão de cinco pontos para a presença de sintomatologia depressiva. Na avaliação da função cognitiva foi utilizada a escala Short Portable Mental Status Questionnaire (SPMSQ), de Pfeiffer (1975).

Após essa etapa, foi feito um estudo-piloto para testagem e calibração do entrevistador e, em seguida, iniciada a coleta dos dados.

Terminada a coleta, os dados foram introduzidos em um banco de dados do programa estatístico SPSS (versão 10.0). A análise foi realizada valendo-se de abordagens de estatística descritiva e outra analítica. Na abordagem descritiva foi feita a distribuição de freqüências absolutas e relativas para variáveis categóricas e médias com \pm para variáveis contínuas.

$\mathrm{Na}$ abordagem analítica foi realizada análise bivariada utilizando o teste do qui-quadrado de Pearson $\left(\chi^{2}\right)$, para se observarem as possíveis associações existentes entre as variáveis independentes e as dependentes, com cálculo das odds ratios (OR) brutas. Em seguida foi realizada análise multivariada, por regressão logística binária, utilizando análise hierarquizada. Mediante a estratégia estabelecida de associações entre as dimensões estudadas (sociodemográficas, de saúde física, capacidade funcional e neuropsiquiátricas), foram elaborados três modelos explicativos de regressão logística binária, introduzindo as variáveis em forma de blocos, permanecendo no modelo subseqüente apenas aquelas que tiveram significância estatística $(p<0,05)$ no modelo anterior. $O$ critério de saída para todas as variáveis introduzidas em cada modelo foi $p<0,1$. Ao final, chegou-se a 
um modelo final de regressão com apenas as variáveis de maior significância estatística. 0 método adotado para introdução das variáveis nos modelos foi o backward stepwise. Considerou-se um nível de significância $p<0,05$ e intervalo de confiança (IC) de 95\%, com cálculo das OR ajustadas.

\section{Resultados}

Um total de 310 idosos residentes na zona urbana do município de Santa Cruz foi avaliado, o que correspondeu a $10,09 \%$ da população de 3.070 idosos cadastrados no SIAB do
Departamento de Informação e Informática do Sistema Único de Saúde (SUS), Ministério da Saúde (SIAB/DATASUS/MS) em dezembro de 2001, dos quais $128(41,3 \%)$ pertenceram ao bairro Paraíso, $91(29,4 \%)$ ao CCM e $91(29,4 \%)$ ao Centro. A idade variou de 60 a 99 anos, com média de $73,7 \pm 9,03$. A distribuição das variáveis sociodemográficas, de saúde física e capacidade funcional pelas zonas estão contidas nas Tabelas 1,2 e 3 , respectivamente.

Em relação à presença de sintomatologia depressiva, observou-se uma prevalência de $25,5 \%$ de idosos, sendo 0 bairro Paraíso o local com maior freqüência. 0 déficit cognitivo

Tabela 1. Distribuição das variáveis sociodemográficas pelas zonas do município de Santa Cruz-RN

\begin{tabular}{|c|c|c|c|c|c|c|c|c|}
\hline & \multirow{2}{*}{\multicolumn{2}{|c|}{$\begin{array}{c}\text { Paraíso } \\
n=128\end{array}$}} & \multicolumn{2}{|c|}{$\mathrm{CCM}$} & \multicolumn{2}{|c|}{ Centro } & \multicolumn{2}{|c|}{ Geral } \\
\hline & & & \multicolumn{2}{|c|}{$n=91$} & \multicolumn{2}{|c|}{$n=91$} & \multicolumn{2}{|c|}{$n=310$} \\
\hline & $n$ & $\%$ & $n$ & $\%$ & $n$ & $\%$ & $n$ & $\%$ \\
\hline \multicolumn{9}{|l|}{ Sexo } \\
\hline Masculino & 44 & 34,4 & 32 & 35,2 & 37 & 40,7 & 113 & 36,5 \\
\hline Feminino & 84 & 65,6 & 59 & 64,8 & 54 & 59,3 & 197 & 63,5 \\
\hline \multicolumn{9}{|l|}{ Cor } \\
\hline Branca & 52 & 40,6 & 49 & 53,8 & 57 & 62,7 & 113 & 36,5 \\
\hline Parda & 61 & 47,7 & 49 & 53,8 & 57 & 62,6 & 167 & 53,9 \\
\hline Negra & 15 & 11,7 & 7 & 7,7 & 8 & 8,8 & 30 & 9,7 \\
\hline \multicolumn{9}{|l|}{ Escolaridade } \\
\hline Analfabeto & 64 & 50 & 29 & 31,9 & 30 & 33,3 & 123 & 39,7 \\
\hline Semi-analfabeto & 53 & 41,4 & 46 & 50,5 & 36 & 39,6 & 135 & 43,5 \\
\hline Ensino fundamental & 11 & 8,6 & 12 & 13,2 & 22 & 24,2 & 45 & 14,5 \\
\hline Ensino médio & - & - & 4 & 4,4 & 3 & 3,3 & 7 & 2,3 \\
\hline \multicolumn{9}{|l|}{ Estado civil } \\
\hline Casado/em união & 70 & 54,7 & 47 & 51,6 & 56 & 61,5 & 173 & 55,8 \\
\hline Viúvo & 46 & 35,9 & 31 & 34,1 & 27 & 29,7 & 104 & 33,5 \\
\hline Solteiro & 7 & 5,5 & 3 & 3,3 & 5 & 5,5 & 104 & 33,5 \\
\hline Divorciado/separado & 5 & 3,9 & 10 & 11 & 3 & 3,3 & 18 & 5,8 \\
\hline \multicolumn{9}{|l|}{ Atividade laboral } \\
\hline Agricultor & 119 & 93 & 65 & 71,4 & 63 & 69,2 & 247 & 79,7 \\
\hline Dona-de-casa & - & - & 10 & 11 & 7 & 7,7 & 17 & 5,5 \\
\hline ASG & 4 & 3,1 & 4 & 4,4 & 4 & 4,4 & 12 & 3,9 \\
\hline Outros & 5 & 3,9 & 12 & 13,2 & 17 & 18,7 & 34 & 10,9 \\
\hline \multicolumn{9}{|c|}{ Atividades nas horas livres } \\
\hline Sim & 42 & 32,8 & 56 & 61,5 & 51 & 56 & 149 & 48,1 \\
\hline Não & 86 & 67,2 & 35 & 38,5 & 40 & 44 & 161 & 51,9 \\
\hline \multicolumn{9}{|c|}{$\begin{array}{l}\text { Tamanho da família } \\
\text { ( } \mathrm{n}^{0} \text { de moradores do domicílio) }\end{array}$} \\
\hline Um & 8 & 6,3 & 6 & 6,6 & 6 & 6,6 & 20 & 6,5 \\
\hline Dois & 37 & 28,9 & 24 & 26,4 & 28 & 30,8 & 89 & 28,7 \\
\hline Mais de dois & 83 & 64,8 & 61 & 67 & 57 & 62,6 & 201 & 64,8 \\
\hline
\end{tabular}

ASG = auxiliar de serviços gerais. 
Tabela 2. Distribuição das variáveis de saúde física pelas zonas do município de Santa Cruz-RN

\begin{tabular}{|c|c|c|c|c|c|c|c|c|}
\hline & \multirow{2}{*}{\multicolumn{2}{|c|}{$\begin{array}{l}\text { Paraíso } \\
n=128\end{array}$}} & \multirow{2}{*}{\multicolumn{2}{|c|}{$\begin{array}{l}\text { CСM } \\
n=91\end{array}$}} & \multirow{2}{*}{\multicolumn{2}{|c|}{$\begin{array}{l}\text { Centro } \\
n=91\end{array}$}} & \multirow{2}{*}{\multicolumn{2}{|c|}{$\begin{array}{c}\text { Geral } \\
n=310\end{array}$}} \\
\hline & & & & & & & & \\
\hline & $n$ & $\%$ & $n$ & $\%$ & $n$ & $\%$ & $n$ & $\%$ \\
\hline \multicolumn{9}{|c|}{ Percepção de saúde } \\
\hline Ótima & 3 & 2,3 & 8 & 8,8 & 10 & 11 & 21 & 6,8 \\
\hline Boa & 54 & 42,2 & 30 & 33 & 38 & 41,8 & 122 & 39,4 \\
\hline Ruim & 49 & 38,3 & 29 & 31,9 & 21 & 23,1 & 99 & 31,9 \\
\hline Péssima & 11 & 8,6 & 10 & 11 & 12 & 13,2 & 33 & 10,6 \\
\hline NS/NR & 11 & 8,6 & 14 & 15,4 & 12 & 13,2 & 35 & 11,3 \\
\hline \multicolumn{9}{|c|}{ Presença de patologia } \\
\hline $\operatorname{Sim}$ & 125 & 97,7 & 82 & 90,1 & 75 & 82,4 & 282 & 91 \\
\hline Não & 3 & 2,3 & 9 & 9,9 & 16 & 17,6 & 28 & 9 \\
\hline \multicolumn{9}{|c|}{ Diabetes mellitus } \\
\hline $\operatorname{Sim}$ & 24 & 18,8 & 12 & 13,2 & 11 & 12,1 & 47 & 15,2 \\
\hline Não & 104 & 81,3 & 79 & 86,8 & 80 & 87,9 & 263 & 84,8 \\
\hline \multicolumn{9}{|l|}{ HAS } \\
\hline Sim & 70 & 54,7 & 40 & 44,4 & 35 & 38,5 & 145 & 46,8 \\
\hline Não & 58 & 45,3 & 51 & 56 & 56 & 61,5 & 165 & 53,2 \\
\hline \multicolumn{9}{|l|}{ AVC } \\
\hline Sim & 15 & 11,7 & 4 & 4,4 & 7 & 7,7 & 26 & 8,4 \\
\hline Não & 113 & 88,3 & 87 & 95,6 & 84 & 92,3 & 284 & 91,6 \\
\hline \multicolumn{9}{|l|}{ Obesidade } \\
\hline Sim & 18 & 14,1 & 9 & 9,9 & 8 & 8,8 & 35 & 11,3 \\
\hline Não & 110 & 85,9 & 82 & 90,1 & 83 & 91,2 & 275 & 88,7 \\
\hline \multicolumn{9}{|c|}{ Doenças pulmonares } \\
\hline Sim & 17 & 13,3 & 3 & 3,3 & 10 & 11 & 30 & 9,7 \\
\hline Não & 111 & 86,7 & 88 & 96,7 & 81 & 89 & 280 & 90,3 \\
\hline \multicolumn{9}{|c|}{ Fratura de quadril } \\
\hline Sim & 4 & 3,1 & 5 & 5,5 & 3 & 3,3 & 12 & 3,9 \\
\hline Não & 124 & 96,9 & 86 & 94,5 & 88 & 96,7 & 298 & 96,1 \\
\hline \multicolumn{9}{|l|}{ Reumatismo } \\
\hline Sim & 83 & 64,8 & 42 & 46,2 & 40 & 44 & 165 & 53,2 \\
\hline Não & 45 & 35,2 & 49 & 53,8 & 51 & 56 & 145 & 46,8 \\
\hline \multicolumn{9}{|l|}{ Déficit visual } \\
\hline Sim & 108 & 84,4 & 71 & 78 & 64 & 70,3 & 243 & 78,4 \\
\hline Não & 20 & 15,6 & 20 & 22 & 27 & 29,7 & 67 & 21,6 \\
\hline \multicolumn{9}{|c|}{ Déficit auditivo } \\
\hline Sim & 50 & 39,1 & 33 & 36,3 & 37 & 40,7 & 120 & 38,7 \\
\hline Não & 78 & 60,9 & 58 & 63,7 & 54 & 59,3 & 190 & 61,3 \\
\hline \multicolumn{9}{|l|}{ Câncer } \\
\hline Sim & 2 & 1,6 & 4 & 4,4 & 3 & 3,3 & 9 & 2,9 \\
\hline Não & 126 & 98,4 & 87 & 95,6 & 88 & 96,7 & 301 & 97,1 \\
\hline \multicolumn{9}{|c|}{ Quantidade de medicamentos } \\
\hline Nenhum & 57 & 44,5 & 49 & 53,8 & 60 & 65,9 & 166 & 53,5 \\
\hline Um & 34 & 26,6 & 18 & 19,8 & 10 & 11 & 62 & 20 \\
\hline Dois & 15 & 11,7 & 16 & 17,6 & 15 & 16,5 & 46 & 14,8 \\
\hline Mais de dois & 22 & 17,2 & 8 & 8,8 & 6 & 6,6 & 36 & 11,7 \\
\hline \multicolumn{9}{|c|}{$\mathrm{N}^{0}$ de internações no último ano } \\
\hline Nenhuma & 116 & 90,6 & 83 & 91,2 & 83 & 91,2 & 282 & 91 \\
\hline Uma ou mais & 12 & 9,4 & 8 & 8,8 & 8 & 8,8 & 28 & 9 \\
\hline
\end{tabular}

NS/NR = não sabe ou não respondeu. 
Tabela 3. Distribuição das variáveis neuropsiquiátricas segundo as zonas do município de Santa Cruz-RN

\begin{tabular}{|c|c|c|c|c|c|c|c|}
\hline \multicolumn{2}{|c|}{ Paraíso } & \multicolumn{2}{|c|}{$\mathrm{CCM}$} & \multicolumn{2}{|c|}{ Centro } & \multicolumn{2}{|c|}{ Geral } \\
\hline & & & & & & & \\
\hline$n$ & $\%$ & $n$ & $\%$ & $n$ & $\%$ & $n$ & $\%$ \\
\hline
\end{tabular}

\begin{tabular}{lcccccccc}
\hline Função cognitiva & & & & & & & & \\
Com alteração & 46 & 35,9 & 20 & 22 & 17 & 18,7 & 83 & 26,8 \\
Sem alteração & 82 & 64,1 & 71 & 78 & 74 & 81,3 & 227 & 73,2 \\
Escore GDS-15 & & & & & & & & \\
$>5$ & 41 & 32 & 17 & 18,7 & 21 & 23,1 & 79 & 25,5 \\
$<5$ & 79 & 61,7 & 70 & 76,9 & 67 & 73,6 & 216 & 69,7 \\
Não respondeu & 8 & 6,3 & 4 & 4,4 & 3 & 3,3 & 15 & 4,8 \\
\hline
\end{tabular}

foi encontrado em $26,8 \%$ dos entrevistados (Tabela 4). Para a realização das análises bivariada e multivariada foram feitos reagrupamentos em algumas variáveis, com o objetivo de melhor executá-las. Isso ocorreu levando em conta a distribuição de freqüência e modelos encontrados na literatura. Também foram retirados da análise todos os sujeitos que não responderam ao teste $(n=15)$, uma vez que eram, na sua maioria, pessoas com elevado grau de déficit cognitivo ou distúrbio de comunicação (auditivo ou da fala).

Entre as variáveis sociodemográficas, apenas a escolaridade $(p=0,038)$ e a ocorrência de atividades nas horas livres $(p=0,003)$ tiveram significância estatística com a presença de sintomatologia depressiva. Em relação às variáveis de saúde física, encontrou-se associação com presença de sintomatologia depressiva, percepção de saúde atual $(p<0,0001), \operatorname{ABVD}(p<0,0001)$, AIVD $(p<0)$ e número de internações no último ano $(p=0,025)$. Especificamente por patologias, foi encontrada associação com 0 acidente vascular cerebral (AVC) $(p=0,031)$ e as doenças pulmonares $(p=0,021)$. Por fim, a função cognitiva $(p=0,001)$ também teve significância.

$\mathrm{Na}$ análise multivariada, entraram no modelo de regressão apenas as variáveis que tiveram $p<0,05$, e permaneceram com significância estatística as variáveis idade acima de 75 anos ( $p=0,046)$, analfabetismo $(p=0,037)$, má percepção de saúde $(p<0,01)$ e dependência para as AIVD $(p=0,001)$ (Tabela 5).

Tabela 4. Distribuição da avaliação da capacidade funcional segundo as zonas do município de Santa Cruz-RN

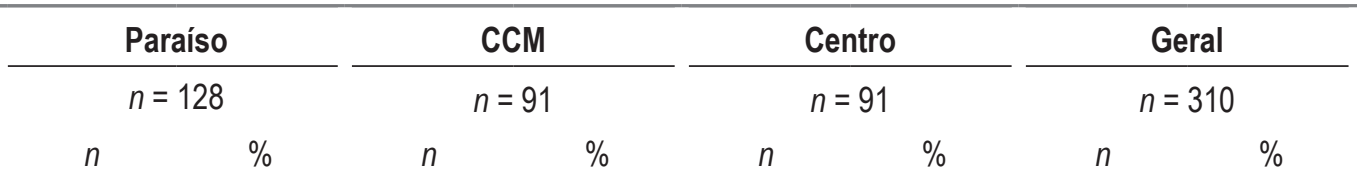

\begin{tabular}{|c|c|c|c|c|c|c|c|c|}
\hline & $n$ & $\%$ & $n$ & $\%$ & $n$ & $\%$ & $n$ & $\%$ \\
\hline \multicolumn{9}{|l|}{ ABVD } \\
\hline Autonomia total & 105 & 82 & 82 & 90,1 & 82 & 90,1 & 269 & 86,8 \\
\hline Dependência leve & 16 & 12,5 & 4 & 4,4 & 5 & 5,5 & 25 & 8,1 \\
\hline Dependência moderada & 4 & 3,1 & 2 & 2,2 & 1 & 1,1 & 7 & 2,3 \\
\hline Dependência grave & - & - & 1 & 1,1 & - & - & 1 & 0,3 \\
\hline Dependência total & 3 & 2,3 & 2 & 2,2 & 3 & 3,3 & 8 & 2,6 \\
\hline \multicolumn{9}{|l|}{ AIVD } \\
\hline Autonomia total & 48 & 37,5 & 51 & 56 & 48 & 52,7 & 147 & 47,7 \\
\hline Dependência leve & 47 & 36,7 & 23 & 25,3 & 23 & 25,3 & 93 & 30 \\
\hline Dependência moderada & 11 & 8,6 & 7 & 7,7 & 12 & 13,2 & 30 & 9,7 \\
\hline Dependência grave & 12 & 9,4 & 2 & 2,2 & 2 & 2,2 & 16 & 5,2 \\
\hline Dependência total & 10 & 7,8 & 8 & 8,8 & 6 & 6,6 & 24 & 7,7 \\
\hline
\end{tabular}


Tabela 5. Resultado de análise multivariada para associação das variáveis independentes com a alteração no equilíbrio dos idosos residentes na comunidade do município de Santa Cruz-RN

\begin{tabular}{|c|c|c|c|c|}
\hline Variáveis & Referência & $p$ & $O R_{\text {austada }}$ & IC 95\% \\
\hline Idade & \multirow{3}{*}{$<75$ anos } & \multirow{3}{*}{0,046} & \multirow{3}{*}{2,73} & \multirow{3}{*}{$1,02-7,33$} \\
\hline$>75$ anos & & & & \\
\hline Escolaridade & & & & \\
\hline $\begin{array}{l}\text { Analfabeto/ } \\
\text { semi- } \\
\text { analfabeto }\end{array}$ & Alfabetizado & 0,037 & 2,19 & $1,05-4,57$ \\
\hline Saúde atual & \multirow{3}{*}{ Ótima } & \multirow{3}{*}{0} & \multirow{3}{*}{6,15} & \multirow{3}{*}{$3,09-13,71$} \\
\hline Péssima & & & & \\
\hline AIVD & & & & \\
\hline Dependência & Independência & 0,001 & 3,72 & $1,7-8,11$ \\
\hline
\end{tabular}

\section{Discussão}

A alta prevalência de sintomatologia depressiva de $25,5 \%$ encontrada no presente estudo foi similar à referida em outros trabalhos (Vea et al., 1999; Gazalle et al., 2004) quando considerados os idosos residentes na comunidade, porém um pouco maior que um recente estudo realizado no Brasil (Barbosa et al., 2005). Um fato que pode favorecer essa alta prevalência diz respeito ao local de realização do estudo, uma vez que o município de Santa Cruz caracteriza-se por ser uma cidade de recursos sociais escassos. Nesse contexto, a depressão pode resultar da incapacidade de uma pessoa lidar com determinados níveis de tensão ambiental. Os fatores externos, como rendimentos, suporte social ou recursos sociais, afetam a capacidade da pessoa em se adaptar aos diversos fatores tensiogênicos (Rautio et al., 2001).

Esse quadro foi muito bem representado com os idosos residentes no bairro Paraíso, que apresentaram os piores resultados $(32 \%)$, demonstrando o importante impacto do ambiente na apresentação dessa enfermidade, uma vez que essa zona compreende a área de menor suporte médico e social, com elevados índices de morbimortalidade, analfabetismo e desemprego. A própria estrutura física do bairro, com ruas não-pavimentadas, esgotos a céu aberto e casas de taipa contribui para a formação de um cenário, por si só, deprimente. Apesar da pequena diferença para as demais regiões da cidade, podemos considerá-lo suficientemente estruturado para mostrar piores indicadores de saúde e qualidade de vida (Costa et al., 2005).

Como resultado, a escassez de uma cobertura social estreita a relação com o envelhecimento acompanhado de doenças, de limitações para o desempenho de atividades cotidianas e de incapacidades definitivas, além de reduzir a oferta de programas de educação em saúde (Feliciano et al., 2004; Kawamoto et al., 2004). Da mesma forma, a baixa renda per capita dos idosos, associada a um suporte médico ineficiente priva-os de uma adequada assistência de saúde, da compra dos medicamentos ou do pagamento da mensalidade do plano de saúde, com reflexos diretos no aumento da chance de surgimento de quadro depressivo, uma vez que a renda é um dos elementos essenciais para a preservação da autonomia e para a manutenção ou a recuperação da saúde (Costa et al., 2001). $\mathrm{Na}$ grande maioria, são idosos cadastrados nos programas de transferência de renda do governo federal, cuja média de benefícios é de um salário mínimo.

As variáveis idade acima de 75 anos, analfabetismo, má percepção de saúde e dependência para AIVD foram as que permaneceram no modelo de regressão logística, com associação independente para a presença de sintomatologia depressiva. Esse fato indica a presença de variáveis dos três blocos de fatores de exposição analisados, o que reforça a íntima dependência da depressão com as dimensões sociodemográfica, de saúde física e capacidade funcional.

O impacto da idade na ocorrência de depressão pode ser compreendido pela própria característica do processo de envelhecimento, pois há diminuição na qualidade e na quantidade de informações necessárias para um controle emocional eficiente (erosão afetiva). Além disso, com o passar dos anos, eventos relacionados a perdas, como comprometimento da saúde, morte de familiares, perda das relações sociais, do trabalho e do prestígio social, diminuição do sono, perda do prazer nas atividades habituais e sexuais, favorecem o surgimento daquela patologia (Mehta et al., 2002).

A baixa escolaridade também é considerada um importante fator de risco associado à depressão (Kulaksizoglu, 2005). A educação é um dos principais critérios de avaliação do nível socioeconômico de uma população, outra importante variável, como citado anteriormente. Desta forma, a alta prevalência de analfabetos em Santa Cruz, em associação ao suporte social inadequado, tende a agravar os efeitos negativos da doença e ainda dificultar 0 acesso aos métodos terapêuticos, sendo um fator de limitação para a sobrevivência e para a qualidade de vida (Feliciano et al., 2004).

Apesar de sua subjetividade, a percepção de saúde de um indivíduo, ou seja, a forma como ele se vê em relação ao seu estado geral, incluindo as dimensões biológica, psíquica e social, associa-se intimamente aos quadros depressivos. Uma vez que elementos como perda das funções cognitiva, sexual e laboral, diminuição das relações sociais, sentimento de invalidez, entre outros, influenciam simultaneamente tanto a percepção de saúde como o surgimento de depressão, é justificável a associação entre ambas (Costa et al., 2005). Esse indicador de saúde relaciona-se com sintomas somáticos, principalmente as enfermidades crônicas e invalidantes, que, ao se agravarem, repercutem diretamente na falta de motivação, no aparecimento de sentimentos depressivos e na limitação funcional.

Nas atividades instrumentais, por se tratar de funções que requerem maior elaboração e coordenação, há maior probabilidade de qualquer alteração mínima já constituir um fator para o desenvolvimento de alguma deficiência e com isso gerar um sentimento negativo, que pode levar a um episódio depressivo. 0 risco aumentado de depressão nas pessoas idosas com incapacidade torna essencial identificar os fatores que estão causando a limitação. Apatia, redução do prazer nas atividades sociais e retardo psicomotor diminuem a capacidade 
de execução das AIVD, fazendo com que tarefas aparentemente simples passem a exigir quantidades excessivas de energia, tornando-se extremamente difíceis (Steffens et al., 2005).

Dessa forma, as limitações funcionais configuram-se como os primeiros indicativos de um possível quadro de institucionalização e surgimento de síndromes geriátricas, entre outras complicações, como a depressão. Além disso, a visão de uma provável invalidez e o isolamento social podem contribuir para o surgimento e a persistência desse quadro (Menchetti et al., 2001).

Apesar de algumas limitações, principalmente pelo desenho do estudo, mas considerando a consistência das associações encontradas, é possível afirmar que aquelas variáveis são potenciais fatores associados ao aparecimento e ao agravamento dos quadros depressivos na população idosa. Existe uma ampla gama de fatores que contribuem para a saúde dos idosos ou os colocam em situações de risco. Esses determinantes raramente existem separadamente e, por isso mesmo, poucas vezes se beneficiam de soluções unidimensionais. Assim, é necessária a colaboração de múltiplos setores e parceiros para atender a fatores de risco inter-relacionados e salvaguardar aqueles que promovem o envelhecimento ativo.

Em termos de ações de combate a esse mal, as políticas de assistência ao idoso podem ser orientadas pelos achados de estudos dessa natureza. Mesmo considerando a idade uma variável imutável, a percepção de saúde, a manutenção da capacidade funcional e a melhor oferta de programas de alfabetização podem ser instituídas nas políticas de assistência ao idoso.

\section{Conclusão}

As variáveis idade acima de 75 anos, analfabetismo, má percepção de saúde e dependência para AIVD estiveram associadas de forma independente à presença de sintomatologia depressiva nos idosos da nossa população. Diante do exposto, a partir da metodologia empregada e dos resultados obtidos, foi possível alcançar os objetivos propostos e fazer esclarecimentos pertinentes à saúde dos idosos do município de Santa Cruz. Em virtude das limitações do estudo, merece ser considerada a hipótese de uma possível generalização dos resultados a outras comunidades de mesmo nível socioeconômico.

\section{Referências}

Barbosa AR, Souza JM, Lebrao ML, Laurenti R, Marucci MFN. Functional limitations of Brazilian elderly by age and gender differences: data from SABE survey. Cad Saude Publica, 21: 1177-85, 2005.

Barcia D, Alcantara AG. Depresión en el anciano. Aspectos etiológicos y clínicos. Actas Esp Psiquiatr, 28: 3-9, 2000.

Chattat R, Ellena L, Cucinotta D, Savarani G, Mucciarelli G. A study on the validity of different short versions of the geriatric depression scale. Arch Gerontol Geriatr, 7: 81-6, 2001.

Costa MFL, Firmo JOA, Uchoa E. Differences in self-rated health among older adults according to socioeconomic circumstances: the Bambui Health and Aging Study. Cad Saude Publica, 21: 830-9, 2005.

Costa MFL, Guerra HL, Firmo JS, Uchoa E. Projeto Bambuí: um estudo epidemiológico de características sociodemográficas, suporte social e indicadores de condição de saúde dos idosos em comparação aos adultos jovens. Informe Epidemiológico do SUS, 4: 147-61, 2001.

Feliciano AB, Moraes SA, Freitas ICM. O perfil do idoso de baixa renda no município de São Carlos, São Paulo, Brasil: um estudo epidemiológico. Cad Saude Publica, 20: 1575-85, 2004.

Gazalle FK, Lima MS, Tavares BF, Hallal PC. Sintomas depressivos e fatores associados em população idosa no Sul do Brasil. Rev Saude Publica, 38: 365-71, 2004.
Guccione AA. Fisioterapia Geriátrica. 2 ed. Rio de Janeiro: Guanabara Koogan, 2002.

Katz S, Ford AB, Moskowitz RW, Jackson BA, Jaffe MW. The index of $A D L:$ a standardized measure of biologic and psychosocial function. JAMA, 185: 914-9,1963.

Kawamoto R, Yoshida O, Oka Y. Factors related to functional capacity in community-dwelling elderly. Geriatrics and Gerontology International, 4: 105-10, 2004.

Kulaksizoglu IB, et al. Unrecognized depression in community-dwelling elderly persons in Istanbul. Int Psychogeriatr, 17: 303-12, 2005.

Lawton MP, Brody EM. Assessment of older people: self-maintaining and instrumental activities of daily living. Gerontologist, 9: 179-86, 1969.

Menchetti M, Fava C, Berardi D. Disability associated with depressive symptoms in elderly primary care attenders. Arch Gerontol Geriatr, 7: 261-6, 2001.

Mehta KM, et al. Cognitive impairment, depressive symptoms and functional decline in older people. Journal of American Geriatrics Society, 50: 1045-50, 2002.

Pfeiffer, E. A short portable mental status questionnaire for the assessment of organic brain deficit in elderly patients. J Am Geriatr Soc, 23: 43341, 1975.

Rautio N, Heihhinen E, Heihhinen RL. The association of socio-economic factors with physical and mental capacity in elderly men and women. Arch Gerontol Geriatr, 33: 163-78, 2001. 
Rozenthal M, Laks J, Engelhardt E. Aspectos neuropsicológicos da depressão. Rev Psiquiatr Rio Gde Sul, 26: 204-12, 2004.

Shah A, Herber TR, Lewis S, Mahendran R, Platt J, Bhattacharyya B. Screening for depression among acutely ill geriatric inpatients with a short geriatric depression scale. Age Ageing, 26: 217-21, 1997.

Spear J. Por quanto tempo os idosos devem tomar antidepressivos para evitar recaídas? Rev Bras Psiquiatr, 20: 70-3, 2002.
Snowdon J. How high is the prevalence of depression in old age? Rev Bras Psiquiatr, 24: 42-7, 2002.

Steffens DC, et al. Biological and social predictors of long-term geriatric depression outcome. Int Psychogeriatr, 17: 41-56, 2005.

Vea HB, Fente AF, Gras OT, Jiménez JM. Prevalencia de discapacidad física en ancianos del municipio Playa. 1996 (Parte I). Revista Cubana de Salud Publica, 25: 16-29, 1999. 